\section{Nimmt der Anteil junger Schlaganfallpatienten zu?}

\section{Die Inzidenz des Schlaganfalls ist in hochentwickelten westlichen Ländern seit vielen Jahren rückläu- fig. Eine regionale Studie aus den USA liefert jetzt Hinweise, dass die Schlaganfallinzidenz bei jüngeren Menschen möglicherweise zunimmt.}

- In der bevölkerungsbezogenen Beobachtungsstudie wurden seit 1993 in regelmäßigen Abständen alle Schlaganfälle erfasst, die in der Bevölkerung der Region Greater Cincinnati/Northern Kentucky der USA auftraten. Hierzu wurden Behandlungsdaten von regionalen Akutkrankenhäusern, ambulanten Gesundheitszentren, Hausarztpraxen und Pflegeheimen sowie Daten der regionalen Todesursachenregister herangezogen. Berechnet wurden die Inzidenzraten pro 100000 Einwohner pro Jahr für 1993/94, 1999 und 2005.

Während der Beobachtungszeit von zwölf Jahren sank das durchschnittliche Schlaganfalls signifikant von 71,2 Jahren in den Jahren 1993/94 auf 69,2 Jahre im Alter beim Auftreten eines ersten
Jahr 2005 ( $\mathrm{p}<0,0001)$. Gleichzeitig kam es zu einem Anstieg des Anteils von Schlaganfällen bei jüngeren Erwachsenen im Alter von 20 bis 54 Jahren: 1993/94 traten 12,9\% aller erstmaligen Schlaganfälle in dieser Altersgruppe auf, 2005 waren es dagegen schon $18,6 \%$ aller Schlaganfälle ( $\mathrm{p}=0,002)$. Dabei stieg die Inzidenzrate von 1993/94 bis 2005 von 26 auf 48/100000 bei Weißen und von 83 auf 128/100 000 bei Schwarzen. In den höheren Altersgruppen waren dagegen fallende Inzidenzraten zu beobachten.

Daten des US-amerikanischen nationalen Gesundheitssurveys NHANES zufolge haben während des gleichen Zeitraums die Prävalenzen von Hyperlipidämie, Diabetes und Adipositas in der Bevölkerung signifikant zugenommen.

- B. M. Kissela et al.

Age at stroke: Temporal trends in stroke incidence in a large, biracial population. Neurology 2012; 79: 1781-1787
Kommentar

Fortschritte in der Prävention und Akuttherapie haben in den letzten Jahrzehnten zu kontinuierlich fallenden Inzidenz- und Mortalitätsraten des Schlaganfalls geführt. Aufgrund des demografischen Wandels mit der Alterung der Bevölkerung wird in den kommenden Jahren jedoch trotz insgesamt fallender Raten mit einem Anstieg der Gesamtzahl an Schlaganfällen in den meisten Ländern gerechnet. Die beobachtete Absenkung des Erkrankungsalters und Zunahme des Anteils von Schlaganfällen bei jüngeren Personen hätte im Rahmen des demografischen Wandels eine ebenso hohe Relevanz. Angesichts einer kontinuierlich steigenden Lebenserwartung könnten die individuellen und gesellschaftlichen Belastungen durch Krankheitsfolgen wie Behinderung und Pflegeabhängigkeit zunehmen, wenn betroffene Personen früher erkranken und länger überleben würden.
Als mögliche Erklärung der Verschiebungen in der Altersstruktur bei Schlaganfallpatienten stellen die Autoren einen Zusammenhang mit der zeitgleich angestiegenen Prävalenz maßgeblicher vaskulärer Risikofaktoren wie Diabetes, Adipositas und Hypercholesterinämie in den USA her. Hieraus ergibt sich erneut die vordringliche Bedeutung präventiver Maßnahmen. Einschränkend muss angemerkt werden, dass es sich um die Ergebnisse einer regionalen Studie handelt, die in anderen Regionen und Ländern reproduziert werden müssen. Zudem kann nicht ausgeschlossen werden, dass vermehrte Diagnostik und verbesserte Aufmerksamkeit für die Erkrankung zu mehr diagnostizierten Schlaganfällen bei jüngeren Personen geführt haben.

M. BuSCH =

\section{Sie lesen Qualität}

Damit das auch so bleibt, befragen wir Sie in Kooperation mit tns infratest in den nächsten Wochen.

\section{Ihr Urteil ist uns wichtig. Bitte nehmen Sie teil!}

